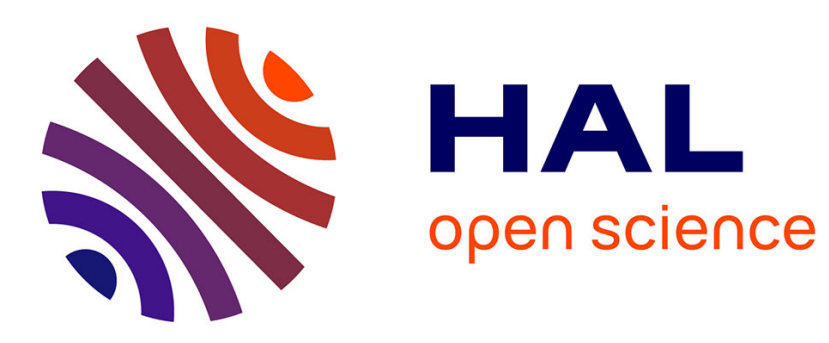

\title{
The Serial Order of Response Units in Word Production: The Case of Typing.
}

\author{
Michele Scaltritti, Marieke Longcamp, F.-Xavier Alario
}

\section{To cite this version:}

Michele Scaltritti, Marieke Longcamp, F.-Xavier Alario. The Serial Order of Response Units in Word Production: The Case of Typing.. Journal of Experimental Psychology: Learning, Memory, and Cognition, 2018, 30 (11), pp.1620-1629. 10.1037/xlm0000494 . hal-01761344

\section{HAL Id: hal-01761344 \\ https://hal.science/hal-01761344}

Submitted on 9 Apr 2018

HAL is a multi-disciplinary open access archive for the deposit and dissemination of scientific research documents, whether they are published or not. The documents may come from teaching and research institutions in France or abroad, or from public or private research centers.
L'archive ouverte pluridisciplinaire HAL, est destinée au dépôt et à la diffusion de documents scientifiques de niveau recherche, publiés ou non, émanant des établissements d'enseignement et de recherche français ou étrangers, des laboratoires publics ou privés. 
(C) 2017, American Psychological Association. This paper is not the copy of record and may not exactly replicate the final, authoritative version of the article. Please do not copy or cite without authors permission. The final article will be available, upon publication, via its DOI: $10.1037 / \mathbf{x l m 0 0 0 0 4 9 4}$

The Serial Order of Response Units in Word Production: The Case of Typing

\author{
Michele Scaltritti ${ }^{1}$, Marieke Longcamp ${ }^{2}$, F.-Xavier Alario ${ }^{1}$ \\ 1. Aix Marseille Univ, CNRS, LPC, Marseille, France \\ 2. Aix Marseille Univ, CNRS, LNC, Marseille, France
}

WORD COUNT: 4,933 (including references, excluding abstract, footnotes, tables, figures)

\author{
Author Note
}

Michele Scaltritti, and F.-Xavier Alario, Aix Marseille Univ, CNRS, LPC, Marseille, France. Marieke Longcamp, Aix Marseille Univ, CNRS, LNC, Marseille, France.

Michele Scaltritti is now at Dipartimento di Psicologia e Scienze Cognitive, Università degli Studi di Trento, Italy.

This work has been carried out within the Brain and Language Research Institute. It was supported by grants ANR-16-CONV-0002 (ILCB), ANR-11-LABX-0036 (BLRI) and ANR-11-IDEX-0001-02 (A*MIDEX).

Correspondence concerning this article should be addressed to: F.-Xavier Alario LPCUMR 7290, Aix Marseille Univ, CNRS, 3 Place Victor Hugo 13100 - Marseille Cedex 3, France, Phone: +33 4135509 72. e mail: francois-xavier.alario@univ-amu.fr 


\begin{abstract}
The selection and ordering of response units (phonemes, letters, keystrokes) represents a transversal issue across different modalities of language production. Here, the issue of serial order was investigated with respect to typewriting. Following seminal investigations in the spoken modality, we conducted an experiment where participants typed as many times as possible a pair of words during a fixed time-window. The two words either shared their first two keystrokes, the last two ones, all the keystrokes, or were unrelated. Fine-grained performance measures were recorded at the level of individual keystrokes. In contrast with previous results from the spoken modality, we observed an overall facilitation for words sharing the initial keystrokes. In addition, the initial overlap briefly delayed the execution of the following keystroke. The results are discussed with reference to different theoretical perspectives on serial order, with a particular attention to the competing accounts offered by position coding models and chaining models. Our findings point to potential major differences between the speaking and typing modalities in terms of interactive activation between lexical and response units processing levels.
\end{abstract}

Keywords: language production; motor control; typing; serial order 


\section{Introduction}

The selection and ordering of response units (phonemes, letters, keystrokes, etc.) represents a transversal issue across different modalities of language production such as speaking, writing, typing, or signing. In spoken production, previous research has clarified the dynamics of serial order using a variety of form priming experiments (for an overview, see Introduction in Damian and Dumay, 2009). Under the assumption that response units activated by the prime remain at least partially active during target processing, differences in priming effects as a function of the position of the units being primed may reveal the dynamics regulating the computation of the units' serial order.

For example, in Sevald and Dell's (1994) seminal experiments, participants were given a fixed amount of time to repeat pairs or quadruplets of words as many times as they could. Mean production times (i.e. trial duration divided by the number of responses produced) were longer for words sharing initial phonemes, and shorter for words sharing final phonemes: interference for initial overlap vs. facilitation for final overlap. According to the authors' serial phonological competition model, shared phonemes reactivate previously produced lexical representation and their corresponding phonemes, because of the interactive activation between the lexical and phonological levels. As phonemes are serially processed, the initial overlap (e.g., in stab-stop) triggers competition for the selection of the following phonemes ( $a b$ or $o p$ ) by miscuing the system to select those belonging to the other word ( $o p$ or $a b)$. This dynamic is absent when the words share final phonemes, as there are no phonemes to miscue after the overlap. In this latter condition, the system may just benefit from facilitation stemming from the repetition of segments (see also O'Seaghdha \& Marin, 2000; Watson, Buxo-Lugó, \& Simmons, 2015).

A comparable approach has been recently implemented to investigate serial ordering in typing. Snyder and Logan (2014) conducted a series of experiments where a prime word was first displayed, followed by a target to be typed. When the prime and target involved the same keystrokes in different orders (e.g., ocean-canoe) performance was not better than in an unrelated condition (e.g., gulps-canoe). Hence, the units' identity was not primed irrespective of their position. A reliable priming effect on response times (RTs) was found for pairs sharing the two initial keystrokes (e.g., stab-stop), but not for pairs sharing the two final keystrokes (e.g., crop-stop): facilitation for initial overlap vs. no effect for final overlap. Additionally, the authors observed that the overlap in initial keystrokes that speeded up the RTs also slowed down response execution by lengthening the interkeystroke interval (IKI) following the overlap ( $t$-o in stop). In their interpretation, the prime (stab) would activate a serial association $(s-t)$ that is shared with the target word, but also the following one $(t-a)$, which is different from that required by the target ( $t-o$, in stop), thereby yielding an interference effect during response execution.

The investigation of the processing of serial order in speech and typing has thus revealed contrasting patterns of results. This could reflect underlying differences in the processing of serial order across the two modalities, or specific features of the experimental paradigms exploited. In fact, experimental features that are seemingly secondary can impact quite relevant aspects of the results (see Damian \& Dumay, 2009). In speech, whether primes are or are not overtly produced can lead to initial overlap interference vs. facilitation (e.g., O’Seaghdha \& Marin, 2000; Sullivan \& Riffel, 1999; Wheeldon, 2003). In Snyder and Logan (2014) primes were not typed. Also, Snyder and Logan (2014) distinguished measures of retrieval and execution (RTs vs. IKIs), whereas those two aspects were collapsed in Sevald and Dell's (1994) measures of production times.

To clarify these issues, we tested the typing modality with the specific experimental protocol previously used by Sevald \& Dell (1994) in speech. This approach provides a direct 
comparison across language production modalities, allowing to directly assess potential differences in processing the serial order of response units.

\section{Experiment}

We measured typing performance using the continuous word production paradigm (O'Seaghdha \& Marin, 2000; Sevald \& Dell, 1994). We tested different forms of overlap between words, with word pairs that either shared the first two, the final two, or all of their keystrokes, against an unrelated baseline condition.

In line with previous research in spoken language production, we first examined a global measure of performance, namely the number of correct words produced in each trial (O'Seaghdha \& Marin, 2000). To elucidate the origin of any effect detected in the global measure, we then divided the performance within trials in its chronometric components by distinguishing measures of response latency and response execution (Logan \& Crump, 2011). For response latency, we measured the time to initiate the first response (initial reaction time, RT) and the average time elapsing between subsequent words (inter-word intervals). Consistently with extant literature on typing, we assumed that words represent chunking units for response planning, where the system computes motor programs for the whole sequence of movements needed to type the next word. As such, these measures should capture the planning phase of each chunk (word) of the response (e.g., Crump \& Logan, 2010; Logan \& Crump, 2011; Yamaguchi \& Logan, 2014a; 2014b). For response execution, we analyzed the IKIs within the sequences of responses.

Following Snyder and Logan (2014), we should expect words sharing initial keystrokes to display facilitation in terms of response times, inter-word intervals as well as first IKI. We should also observe a selective slowdown for the keystroke following the initial overlap. Importantly, these predicted effects oppose one another and do not allow a straightforward prediction for the global measure of performance (mean production times or number of correct responses). For final keystroke overlap, we should expect no effects on either inter-word intervals nor IKIs. .

If present, this pattern of results would be sharply different from that observed in the same paradigm with spoken responses (Sevald \& Dell, 1994). In this modality, initial overlap yields interference, reducing the number of responses produced in this condition whereas final overlap produces facilitation, thus enhancing the number of responses. Even though Sevald and Dell (1994) did not distinguish between inter-word intervals and word articulatory durations, the effects may concern both measures. Note that other investigations using this paradigm have revealed effects in terms of response duration (Watson et al., 2015). In addition, because the effect of overlapping units is assumed to stem from the interaction between the lexical level and the planning of response units' order, it should affect response latencies.

\section{Method}

Participants. Twelve French native-speakers touch typists participated in the experiment ( 5 females; $\mathrm{M}_{\text {age }}=24.08, \mathrm{SD}_{\text {age }}=3$ ). Typing expertise was assessed using a copy typing test described elsewhere (Pinet, Dubarry, \& Alario, 2016). Participants typed on average 57 words per minute $(\mathrm{SD}=14)$, with $86 \%$ accuracy $(\mathrm{SD}=4)$. Participants read and signed an informed consent document before participation. Participation was compensated with $10 €$ per hour.

Materials. Twenty quadruplets of French words were used (10 with 4-letter words, 10 with 5-letter words). ${ }^{1}$ Items in the quadruplets (e.g., bilan, bigot, roman, robot) were selected so that they could be paired amongst themselves to produce all the experimental conditions. In the initial overlap condition, the words shared the first two keystrokes, and no other keystroke in the same positions (e.g., bilan-bigot, roman-robot). In the final overlap 
condition, words shared the last two keystroke, and no other one in the same position (e.g., bilan-roman, bigot-robot). In the unrelated condition, words did not share any keystroke in the same position (e.g., bilan-robot, bigot-roman). Finally, a repeated condition (e.g., bilanbilan, bigot-bigot, etc.) was also included. In this way, all words could be presented in all conditions. For all conditions except the repeated condition, all pairs were presented twice, once with each possible order (e.g., bilan-bigot, bigot-bilan). In total, 320 experimental pairs were administered to each participant ( 80 trials per condition). Note that the same words were used across the four conditions.

Apparatus and Procedure. The experimental procedure followed the one used in Sevald and Dell (1994). Each trial began with a message asking to press the ENTER key when ready to start. Upon pressing of the ENTER key, the pair of words to be typed was displayed along with a message inviting participant to prepare for typing. After 4,000 ms, the message disappeared, the pair of words remained on the screen, and three auditory warning tones $(417 \mathrm{~Hz})$ were presented, followed by a Go-signal $(833 \mathrm{~Hz})$. All tones lasted $100 \mathrm{~ms}$, and were separated by $400 \mathrm{~ms}$ of silence. Participants were instructed to start typing as soon as they heard the Go-signal and to continue typing the two words in sequence, with the goal to produce as many correct words as possible. After $8,000 \mathrm{~ms}$, a Stop-signal tone $(833 \mathrm{~Hz})$ was presented, signaling that the trial was over (keystrokes typed after the stop-signal were not registered). Participants typed the words separated by a space character, and their entire typed response (i.e., the whole sequence of words) appeared on-line on the screen.

Participants performed the experiment seated in an armchair placed at a distance of $\sim 60 \mathrm{~cm}$ from the computer screen, on which visual stimuli and typed responses were displayed. Tones were presented via earphones and responses were collected from a highspeed DirectIN Keyboard PCB v2010 (Empirisoft) to get keystroke timing data with 1 ms accuracy. Eight practice trials were administered before the beginning of the experiment.

Data Processing. Within each trial, single responses were defined as any sequence of keystrokes occurring between two pressings of the spacebar. Based on this segmentation, we first extracted the number of correct responses produced for each word within each trial. ${ }^{2}$ For the repeated condition, the number of correct responses assigned to the word in the trial was equal to the total number of responses produced in the trial divided by two. In fact, this condition actually included a single word (e.g., bilan-bilan) while the others all included two words (e.g., bilan-bigot). We also coded the accuracy of the responses, to detect potential tradeoffs between the number of responses and the number of errors produced.

In terms of chronometric measures, first we extracted the initial RTs, that is, the time elapsing from the onset of the Go-signal to the first keystroke. Second, we extracted interword intervals, that is the time elapsing between the last keystroke of the previous word and the first keystroke of the current one. In terms of IKIs, we measured the time elapsing between the first and the second keystroke (initial IKI), between the penultimate and the last keystroke (final IKI), from the second and the third keystrokes (second IKI), and from the antepenultimate and the penultimate keystrokes (penultimate IKI) of each word.

For accuracy, inter-word intervals, and for all the IKI measures, we excluded the first correct response for each one of the words within each trial, in order to consider a uniform sample of responses in terms of repetition.

Data Analysis. Data were analyzed with linear mixed-effects models. Except where noted, the maximal random effect structure was fitted (Barr, Levy, Scheepers, \& Tily, 2013). Following Box-Cox tests, measures of RT, inter-word intervals, and IKIs were logtransformed. Accuracy analyses used generalized linear mixed-effects models. All analyses were conducted using the lme4 library (version 4_1.1-12; Bates, Maechler, Bolker, \& Walker, 2015) in R (R Core Team, 2015). The effect of condition was assessed by comparing via log-likelihood test an intercept-only model (including random effects) with a model 
including the fixed effect of condition. In case of a reliable main effect, Bonferroni-corrected pairwise comparisons between conditions were conducted. In the text, we focus on the theoretically relevant comparisons between the unrelated baseline condition and all the other ones, to highlight key differences. Statistical results for the full set of pairwise comparisons are reported in Table 1.

\section{Results}

The overlap condition had a significant effect on the number of correct responses produced within each trial $\left(\chi^{2}[3]=13.25, p=.004\right)$. More words were produced in the initial overlap condition than in the unrelated condition. The other conditions did not differ from the unrelated one. For accuracy analyses, we could only fit models with random intercepts, as more complex structures failed to converge. The effect of condition was significant $\left(\chi^{2}[3]=\right.$ $33.57, \mathrm{p}<.001$ ), with higher accuracy in the initial overlap condition compared to the unrelated one. The other conditions did not differ from the unrelated one. The advantage in the initial overlap conditions in terms of number of responses produced thus did not come at the expense of decreased accuracy (Figure 1). We will now move to chronometric measures to understand where the initial overlap advantage stems from.

A

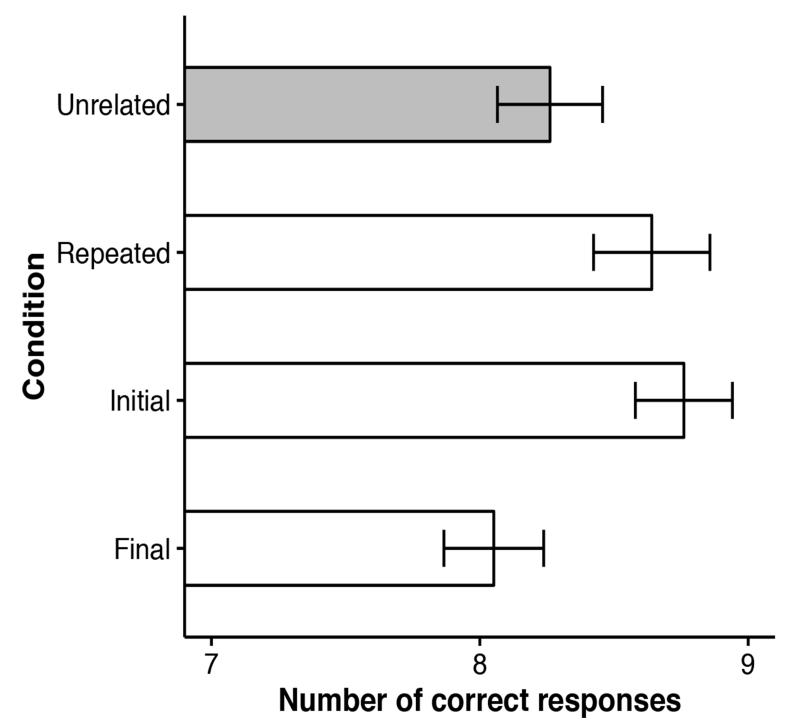

B

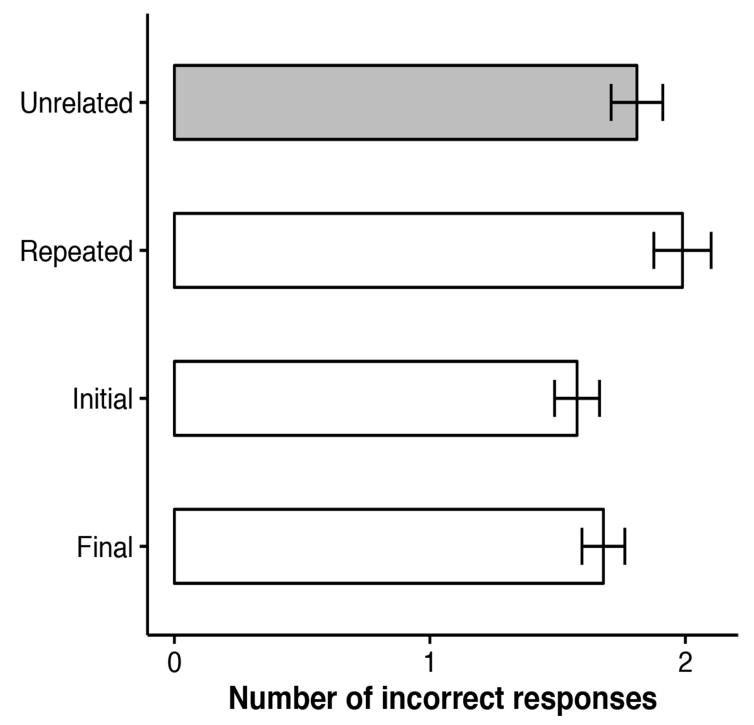

Figure 1. Global performance in the task across conditions. Mean number of correct (A) and incorrect (B) response words produced within trials. The greyed unrelated condition served as baseline. Error bars represent 95\% confidence intervals (adjusted for within-participants variables following Morey, 2008).

For analyses of RTs, we retained just the by-participants random slope as more complex structures failed to converge. The overlap condition did not significantly affect RTs $\left(\chi^{2}[3]=4.49, p=.21\right)$, but it did significantly affect inter-word intervals $\left(\chi^{2}[3]=30.19, p<\right.$ $.001)$, which were shorter in the initial overlap and the repeated conditions, compared to unrelated condition. There was no significant difference between unrelated and the final overlap conditions.

The initial IKI did not reveal any effect of condition $\left(\chi^{2}[3]=1.08, p=.78\right)$. The same was true for the final IKI $\left(\chi^{2}[3]=5.92, p=.12\right)$. Crucially, for the IKI following the initial overlap (i.e., the second IKI), we detected a significant effect of condition $\left(\chi^{2}[2]=21.64, p<\right.$ 
.001 ), with a longer IKI in case of initial overlap compared to both the repeated and the unrelated conditions. Similarly, for the IKI preceding the final overlap (i.e., the penultimate IKI), the effect of condition was significant $\left(\chi^{2}[2]=13.63, p=.002\right)$, with longer IKIs for the final overlap condition compared to both the unrelated and the repeated condition (Table 1). ${ }^{3}$ The results for these chronometric measures are summarized in Figure $2 .{ }^{4}$

In summary, for the initial overlap condition more responses were typed and with a higher accuracy. Here, inter-word intervals were significantly shorter, while a slowdown was detected during response execution, for the IKI following the overlapping keystrokes. For the final overlap, a slowdown was detected for the IKI preceding the overlap. Finally, the full repetition reduced inter-word intervals, but failed to speed-up response execution.

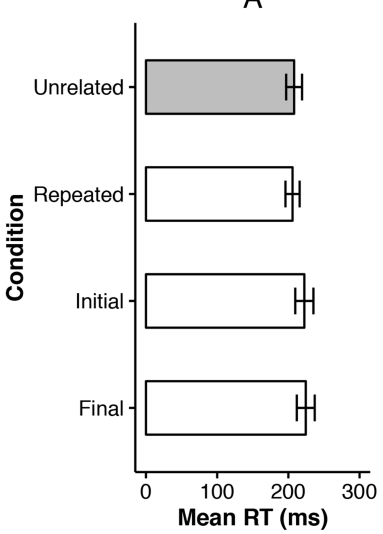

D

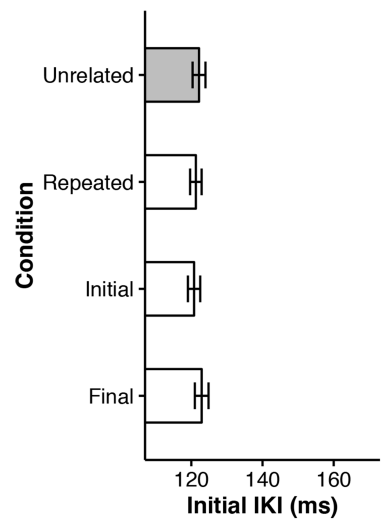

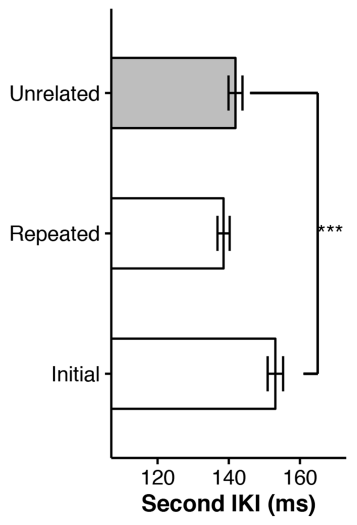

B

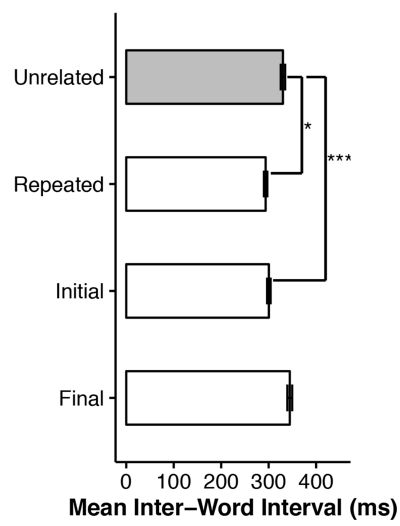

$\mathrm{E}$

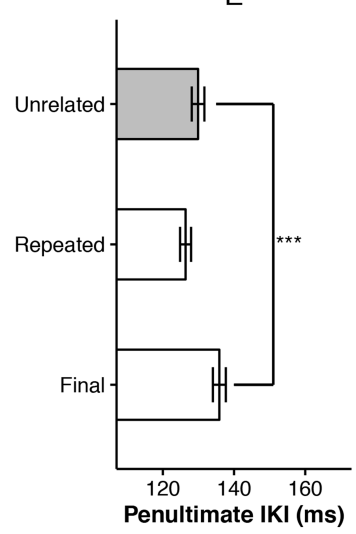

$\mathrm{F}$

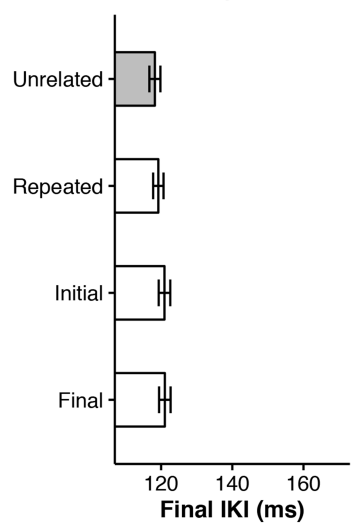

Figure 2. Break down of performance across chronometric indexes as a function of experimental conditions. Mean initial RTs (A), inter-word intervals (B), initial IKI (C), second IKI, (D), penultimate IKI (E), and final IKI (F) as a function of condition. The figure highlights the significant pairwise comparisons against the greyed unrelated conditions that served as baseline. For the full report of the comparisons, see Table 1. Error bars represent 95\% confidence intervals (adjusted for within-participants variables following Morey, 2008) 
Table 1.

Pairwise comparisons between conditions for the different measures considered in the analysis.

\begin{tabular}{|c|c|c|c|c|c|c|c|c|c|c|}
\hline Contrast & \multicolumn{2}{|c|}{ N. Resp } & \multicolumn{2}{|c|}{ IWI } & \multicolumn{2}{|c|}{$\mathrm{ACC}$} & \multicolumn{2}{|c|}{ Second IKI } & \multicolumn{2}{|c|}{ Penultimate IKI } \\
\hline $\mathrm{R}-\mathrm{F}$ & $3.11^{*}$ & $.29(.09)$ & $-5.25 * * *$ & $-.15(.03)$ & -1.02 & $.04(04)$ & - & - & $-2.73^{*}$ & $-.07(.02)$ \\
\hline R - I & -0.60 & $-.06(.10)$ & -0.53 & $-.01(.03)$ & $-5.42 * * *$ & $-.22(.04)$ & $-4.28 * * *$ & $-.10(.02)$ & - & - \\
\hline U - I & $-3.27 * *$ & $-.25(.08)$ & $5.30 * * *$ & $.09(.02)$ & $-4.30 * * *$ & $-.18(.04)$ & $-5.67 * * *$ & $-.07(.01)$ & - & - \\
\hline$U-R$ & -1.69 & $-.19(.11)$ & $2.87 *$ & $.10(.04)$ & .95 & $.04(.04)$ & 1.29 & $.03(.02)$ & 0.92 & $.02(.02)$ \\
\hline
\end{tabular}

Note. N. Resp. = number of correct responses; IWI = inter-word interval, the time elapsing between the last keystroke of a word and the first one of the next word (including spacebar pressing). Second IKI = time elapsing between the second and the third keystroke. Penultimate IKI $=$ time elapsing between the antepenultimate and the penultimate keystroke. Est. = estimated difference; for measures involving time in millisecond, estimates reflect $\log$-transformed values; for accuracy, estimates is in terms of log-odds. $\mathrm{SE}=$ standard error. $\mathrm{I}=$ initial overlap; $\mathrm{F}=$ final overlap; $\mathrm{U}=$ unrelated; $\mathrm{R}=$ repeated. $* * *=p<.001 ; * *=p<.01 ; *=p<.05$. 


\section{General Discussion}

During the continuous typewritten production of word pairs, more words were produced if stimuli shared their initial keystrokes than if they were unrelated. Repeated pairs also produced better performance (shorter inter-word intervals) but that advantage did not significantly culminate in more words being produced overall in that condition. In contrast, an overlap in terms of final keystrokes did not facilitate performance, and did not affect the number of words produced. This pattern of results is the opposite of what has been observed in spoken production (O'Seaghdha \& Marin, 2000; Sevald \& Dell, 1994).

The advantage detected in the initial overlap condition was driven seemingly by interword intervals. A similar advantage was not present in the final overlap condition. This is fully consistent with previous findings by Snyder and Logan (2014), who reported a similar pattern for RTs in the context of a priming experiment. According to those authors, facilitation in the initial overlap condition, paired with the lack of influence exerted by the final overlap, is consistent with a chaining account of serial order. In chaining models, identity and position of response units are jointly represented in a sequence of "left-to-right" directional associations (in language written left to right). Starting from the first item, each element serves as a cue for the following one. In this context, sharing response units in the same position is not sufficient to trigger priming effects. To be effective, recurring units that share common directional associations should also be present within the two sequences. Words with the same initial keystrokes do share some directional associations that can be primed. For example, stab and stop both share the association $s$ - $t$. For sequences overlapping in final keystrokes, the chains of associations leading to the shared keystrokes are different, thus preventing any priming effect. For example, the sequence of keystrokes' associations leading to $o-p$ is different in crop and stop and no priming effect is found (Snyder and Logan, 2014).

Two things are worth noting. First, our results are in clear contrast with respect to those obtained in spoken production using the same paradigm (Sevald \& Dell, 1994), suggesting that a serial competition model may not capture the results in typing, and that the serial order of response units might be processed in different ways across the two modalities. Second, our pattern of results is consistent with the one reported by Snyder and Logan (2014). The authors interpreted their results as consistent with a chaining account. For psycholinguistics, the chaining account represents a major departure from the more traditional position coding perspective, where identity and position of response units are independently represented, and order emerges from the assignment of each unit to a slot within a positional frame (e.g., Dell, 1986; Levelt, Roelofs, \& Meyer, 1999; ShattuckHufnagel, 1979). Do we need to abandon this perspective for typing? In the remainder of this section, we examine this possibility. We also discuss important differences between the two modalities, and further details of our results.

\section{Chaining and Position Coding}

Our results may not definitively discriminate chaining and position coding alternatives. Chaining assumptions computationally implemented in the form of a recurrent network, can in fact also simulate results closer to Sevald and Dell's original ones (Watson et al., 2015). The contrasting effect of the initial and final overlap conditions may thus not be diagnostic of chaining vs. position coding.

Additionally, chaining assumptions have been argued to be challenged by studies that examined the chunking units of typed responses. Yamaguchi and Logan (2014b) examined the contribution of different levels of linguistic units (words, digraphs, and letters) on 
learning new letter-to-key associations. In their experiment, they disrupted established letterto-key associations by switching keyboard locations for a few target letters (i.e., switchedlocation letters). They noted that practicing with sequences containing specific transitions from non-switched to switched letters, as well as from switched to non-switched letters, both produced significant benefits in terms of learning the new letter-to-key associations. According to the authors, these results contradicts serial chaining, as only transitions from non-switched to switched letters are supposed to be effective in these models. However, we would argue that such results may be problematic not only for a serial chaining account, but also for any other account assuming serial processing in the computation of serial order, including those relying on position coding, such as Sevald and Dell's one (see below, discussion of the final overlap effect on the penultimate IKI).

Our pattern of results can also be understood in terms of competitive queuing models, which hold a long tradition in typing (Rumelhart \& Norman, 1982), and which are closer to a position coding perspective. According to these models, response units for a word are activated in parallel within a buffer. Inhibition is applied in a graded fashion across the units, so that more active initial response units are produced before the more inhibited final ones (see Behmer and Crump, 2017, for recent and extensive empirical support). The temporal order of the keystrokes is thus given by the ordering of activation values (Rumelhart \& Norman, 1982). As the initial response units are more active, in our experiment they could have benefited more from the overlap with previous sequences, thus prompting a priming effect on the inter-word interval (i.e., the time needed to initiate the response). In contrast, final response units may have been too inhibited by the competitive queuing to benefit from any overlap with previous sequences. A complication arises when we consider that in these models, typed response units are immediately deactivated. Unless we add the assumption that deactivation does not bring units to zero-level activation, it may be difficult for these models to explain priming effects across different responses.

\section{Typing and Speaking}

A major difference in computing serial order in speaking and typing may lie in the different involvement of higher-level linguistic representations in the two modalities. This is well-motivated by the pattern of errors in the two modalities. During speaking, phonemes involved in slips of the tongue tend to keep their position within the syllable or the word, suggesting that a positional scheme established by the lexical representation constrains the ordering of response units (e.g., Dell, 1986; Shattuck-Hufnagel, 1979). In typing, aspects related to the keyboard layout and to motor patterns tend to have a more noticeable role in shaping errors (e.g., Logan, 1999). Here, the reliance on more abstract linguistic representations might be diminished, and serial order may be established more in terms of motor programs.

Indeed, language and motor processing have been described as two distinct processing loops in the context of typing (Logan \& Crump, 2011). Our recent electrophysiological investigation (Scaltritti, Pinet, Longcamp, \& Alario, 2017) suggested that these two loops may be organized serially (although some linguistic properties seem to affect response execution; Pinet, Ziegler, \& Alario, 2016; Scaltritti, Arfé, Torrance, \& Peressotti, 2016). In the experiment reported here, this functional organization may have prevented any competition stemming from re-activation of previous lexical representations during the planning phase of the responses, thus preventing any interference effect.

\section{Response Execution}


Moving from the planning phase indexed by inter-word intervals, to the phase of response execution indexed by IKIs, we observed that overlapping initial keystrokes between two words delay the execution of the following keystroke. This phenomenon was already highlighted by Snyder and Logan (2014), and considered as evidence that priming from the previous responses affects the phase in which chained motor programs are implemented to perform the keystrokes. Interestingly, this effect bears some resemblance with the serial miscuing dynamic highlighted by Sevald and Dell for spoken language (see the Introduction). In emphasizing left-to-right processing dynamics of serial order, in the form of either directional associative links between motor programs (chaining) or of serial assignments of phonemes (serial phonological competition), both perspectives suggest that initial overlap has detrimental effects on the following response units. In the case of typing, in the initial overlap condition the system may benefit from previously used motor programs in the planning phase, whereas initial overlap would produce interference during the execution of the diverging keystrokes.

Unexpectedly, we also found that for words sharing the final two keystrokes, the penultimate IKI is delayed. This unpredicted observation is hard to reconcile with either the serial phonological competition or the chaining account because the begin-to-end directionality assumed by both model makes the final overlap irrelevant for performance. Snyder and Logan (2014) did not detect this slowdown in their experiment, where primes were not typed. It is thus possible that this unpredicted effect is mostly related to biomechanical and motor aspects recruited during response execution. There is indeed evidence that a given IKI depends not only on the keystrokes that have been typed before ("left-to-right" influence), but also on those that are going to be typed afterwards ("right-toleft influence", e.g., Gentner, 1982). More research is needed that targets the contrast between serial order planning and execution in typing.

The repetition of the same (partial) sequence of keystrokes across words did not speed up the corresponding IKIs. This was true both for the first IKI in the initial overlap condition and, more generally, for the IKIs in the repeated condition. This could reflect a floor effect, whereby expert typists could not further speed up their typing rate, thus failing to highlight any priming during response execution. The repeated condition, however, displays the same advantage as the initial overlap condition in terms of inter-word interval, a result consistent with the idea that overlapping sequences are critical to obtain priming in the planning phase of the response.

\section{Limitations}

One acknowledged limitation of our study is that it was not conceived to tease apart serial ordering in letter vs. keystroke representations. In line with extant literature (Snyder \& Logan, 2014), we interpreted our results in terms of motor programs. However, we cannot definitively ascribe the reported phenomena to the planning of motor programs related to keystrokes, or to a more abstract level in which letter representations are retrieved and ordered. The same might be true for experiments in the spoken modality. Thus, the pattern we outlined can still be deemed informative about the mechanisms underlying serial order across different output modalities, even though the precise level of abstraction at which these mechanisms operate remains to be explored in detail.

\section{Conclusions}

To conclude, typing differs with respect to spoken production in the processing of serial order of response units. A few possibilities are to be considered when trying to understand this major difference. In line with some recent proposals (Synder \& Logan, 2014), 
serial order in typing may rely on chaining rather than on position coding. Furthermore, serial ordering of response units in typing might be less reliant on higher-level linguistic representations. Finally, although "left-to-right" processing dynamics appear to be critical for the planning of the typed responses, they cannot fully account for patterns of response execution. This calls for additional investigations of serial order processes and their link with motor execution of the corresponding sequence of response units.

Overall, our study suggests that we may need to abandon the tempting assumption that the processing of serial order is uniformly achieved across all modalities of language production. 


\section{References}

Barr, D. J., Levy, R., Scheepers, C., \& Tily, H. J. (2013). Random effects structure for confirmatory hypothesis testing: Keep it maximal. Journal of Memory and Language, $68,255-278$.

Bates, D., Maechler, M., Bolker, B., \& Walker, S. (2015). Fitting Linear Mixed-Effects Models Using lme4. Journal of Statistical Software, 67, 1-48.

Behmer, L. P., Jr., \& Crump, M. J. C. (2017). The dynamic range of response set activation during action sequencing. Journal of Experimental Psychology: Human Perception and Performance. Advance online publication. doi: 10.1037/xhp0000335

Crump, M. J. C., \& Logan, G. D. (2010). Hierarchical control and skilled typing: Evidence for word-level control over the execution of individual keystrokes. Journal of Experimental Psychology: Learning, Memory, and Cognition, 36, 1369-1380.

Damian, M. F., \& Dumay, N. (2009). Exploring phonological encoding through repeated segments. Language and Cognitive Processes, 24, 685-712.

Dell, G. S. (1986). A spreading activation theory of retrieval in language production. Psychological Review, 93, 283-321.

Gentner, D. R. (1982). Evidence against a central model of timing in typing. Journal of Experimental Psychology: Learning, Memory, and Cognition, 8, 793-810.

Levelt, W. J. M., Roelofs, A., \& Meyer, A. S. (1999). A theory of lexical access in speech production. Behavioral and Brain Sciences, 22, 1-75.

Logan, F. A. (1999). Errors in copy typewriting. Journal of Experimental Psychology: Human Perception and Performance, 25, 1760-1773.

Logan, G. D., \& Crump, M. J. (2011). Hierarchical control of cognitive processes: The case for skilled typewriting. The Psychology of Learning and Motivation, 54, 1-27.

Morey, R. D. (2008). Confidence intervals for normalized data. A correction to Cousineau (2005). Tutorial in Quantitative Methods for Psychology, 4, 61-64.

Ostry, D. J. (1983). Determinants of interkey times in typing. In W. E. Cooper (Ed.), Cognitive aspects of skilled typewriting (pp. 225-246). New York: Springer-Verlag

O'Seaghdha, P. G., \& Marin, J. W. (2000). Phonological competition and cooperation in form-related priming: sequential and nonsequential processes in word production. Journal of Experimental Psychology: Human Perception and Performance, 26, 57-73.

Pinet, S., Dubarry, A. S., \& Alario, F.-X. (2016). Response retrieval and motor planning during typing. Brain and Language, 159, 74-83.

Pinet, S., Ziegler, J., \& Alario, F.-X. (2016). Typing is writing: Linguistic properties modulate typing execution. Psychonomic Bulletin \& Review, 23, 1989-1906.

$\mathrm{R}$ Core Team (2015). $R$ : A language and environment for statistical computing. R Foundation for Statistical Computing, Vienna, Austria.

Rumelhart, D. E., \& Norman, D. A. (1982). Simulating a skilled typist: A study of skilled cognitive-motor performance. Cognitive Science, 6, 1-36.

Scaltritti, M., Arfé, B., Torrance, M., \& Peressotti, F. (2016). Typing pictures: Linguistic processing cascades into finger movements. Cognition, 156, 16-29. 
Scaltritti, M., Pinet, S., Longcamp, M., \& Alario, F.-X. (2017). On the functional relationship between language and motor processing in typewriting: An EEG study. Language, Cognition and Neuroscience. Advance online publication. doi: 10.1080/23273798.2017.1283427

Sevald, C. A., \& Dell, G. S. (1994). The sequential cuing effect in speech production. Cognition, 53, 91-127.

Shattuck-Hufnagel, S. (1979). Speech errors as evidence for a serial ordering mechanism in sentence production. In W.E. Cooper \& E.C.T Walker (Eds.), Sentence processing : Psycholinguistic studies presented to Merril Garrett (pp. 295-342). Hillsdale, NJ: Erlbaum.

Snyder, K. M., \& Logan, G. D. (2014). The problem of serial order in skilled typing. Journal of Experimental Psychology: Human Perception and Performance, 40, 1697-1717.

Sullivan, M. P., \& Riffel, B. (1999). The nature of phonological encoding during spoken word retrieval. Language and Cognitive Processes, 14, 15-45.

Watson, D. G., Buxó-Lugo, A., \& Simmons, D. C. (2015). The effect of phonological encoding on word duration: Selection takes time. In E. Gibson \& L. Frazier (Eds.), Explicit and implicit prosody in sentence processing (pp. 85-98). Switzerland: Springer International Publishing.

Wheeldon, L. (2003). Inhibitory form priming of spoken word production. Language and cognitive processes, 18, 81-109.

Yamaguchi, M. \& Logan, G. D. (2014a). Pushing typists back on the learning curve: Revealing chunking in skilled typewriting. Journal of Experimental Psychology: Learning, Memory, and Cognition, 40, 592-612.

Yamaguchi, M. \& Logan, G. D. (2014b). Pushing typists back on the learning curve: Contributions of multiple linguistic units in the acquisition of typing skill. Journal of Experimental Psychology: Learning, Memory, and Cognition, 40, 1713-1732. 


\section{Footnotes}

1. A nonword (cavil) was erroneously selected as a stimulus. When running the analyses excluding this item, results did not change.

2. This measure enables us to retain the effect of our experimental manipulation within items, coherently with the experimental design. We also analyzed measures of production time (8,000 divided by the number of correct responses in the trial, as in Sevald and Dell, 1994). There was no significant effect of condition $\left(\chi^{2}[3]=4.39, \mathrm{p}=.22\right)$. This index however is measured on trials (i.e., on word pairs), rather than on single words. This weakens our analysis by making the experimental manipulation a between-item factor. However, if we adopt the same statistical method used by Sevald and Dell (1994), that is a by-participants ANOVA, the effect of condition is actually significant, $\mathrm{F}_{1}[3,33]=3.10, \mathrm{p}<.05, \mathrm{MSE}=$ $4,675.85, \eta_{\mathrm{p}}^{2}=.22$, and mostly driven by the significant difference between the initial overlap and the unrelated conditions $\left(\mathrm{t}_{1}[11]=3.80, \mathrm{p}<.05\right)$.

3. In the analyses of the second IKI, we excluded the final overlap condition. In the analysis of the penultimate IKI, we excluded the initial overlap conditions. This is because for any given four letter word (e.g., clam), the very same IKI (la) reflects the IKI after the initial overlap in the initial overlap condition (e.g., clam-clos), and the IKI before the final overlap in the final overlap condition (e.g., clam-gram).

4. It is interesting to note that IKIs in the middle of the word were overall slower compared to those corresponding to initial and final letters. This reproduces earlier observations (Ostry, 1983) within more "natural" typing tasks such as single word or sentence copy. 